Supplement of Biogeosciences, 18, 729-737, 2021

https://doi.org/10.5194/bg-18-729-2021-supplement

(C) Author(s) 2021. This work is distributed under

the Creative Commons Attribution 4.0 License.

(c) (1)

Supplement of

\title{
Technical note: Inexpensive modification of Exetainers for the reliable storage of trace-level hydrogen and carbon monoxide gas samples
}

Philipp A. Nauer et al.

Correspondence to: Philipp A. Nauer (philipp.nauer@monash.edu) and Perran L. M. Cook (perran.cook@monash.edu)

The copyright of individual parts of the supplement might differ from the CC BY 4.0 License. 
Table S1: Linear regression statistics of gases released from various sealing rubbers, as depicted in Fig. 2. Please refer to Fig. 2 for treatment legend. Significance: $* * * p<0.001 ; * * p<0.01 ; * p<0.05$

\begin{tabular}{|c|c|c|c|c|c|}
\hline \multirow[t]{2}{*}{ Treatment } & \multirow[t]{2}{*}{ Gas } & \multirow{2}{*}{$\begin{array}{l}\text { Slope } \\
\left(p p b h^{-1}\right)\end{array}$} & \multirow{2}{*}{$\begin{array}{l}\text { Std Error } \\
\left(\mathrm{ppb} \mathrm{h}^{-1}\right)\end{array}$} & \multicolumn{2}{|c|}{ Significance } \\
\hline & & & & $\begin{array}{l}\text { Slope vs. } \\
\text { Control }\end{array}$ & $\begin{array}{l}\text { Slope vs. } \\
\text { Reference gas }\end{array}$ \\
\hline UT2 & $\mathrm{CH}_{4}$ & 0.37 & 0.22 & & \\
\hline UT4 & $\mathrm{CH}_{4}$ & 0.54 & 0.40 & & \\
\hline Tex & $\mathrm{CH}_{4}$ & 0.05 & 0.13 & & \\
\hline Tgr & $\mathrm{CH}_{4}$ & 0.94 & 0.14 & $* * *$ & $* * *$ \\
\hline Tbl & $\mathrm{CH}_{4}$ & 0.34 & 0.32 & & \\
\hline FKM & $\mathrm{CH}_{4}$ & 1.69 & 0.12 & $* * *$ & $* * *$ \\
\hline NBR & $\mathrm{CH}_{4}$ & 0.27 & 0.07 & $* *$ & $* * *$ \\
\hline SPA & $\mathrm{CH}_{4}$ & 0.49 & 0.08 & $* * *$ & $* * *$ \\
\hline SPB & $\mathrm{CH}_{4}$ & 0.52 & 0.18 & * & * \\
\hline SSW & $\mathrm{CH}_{4}$ & 0.47 & 0.11 & $* *$ & $* * *$ \\
\hline Con & $\mathrm{CH}_{4}$ & 0.04 & 0.09 & n.a. & \\
\hline Con3 & $\mathrm{CH}_{4}$ & -0.01 & 0.57 & n.a. & \\
\hline UT2 & $\mathrm{CO}$ & 163 & 10.3 & $* * *$ & $* * *$ \\
\hline UT4 & $\mathrm{CO}$ & 322 & 17.0 & $* * *$ & $* * *$ \\
\hline Tex & $\mathrm{CO}$ & 45.0 & 3.37 & $* \star *$ & $* * *$ \\
\hline Tgr & $\mathrm{CO}$ & 17.7 & 0.52 & $* * \star$ & $* * *$ \\
\hline Tbl & $\mathrm{CO}$ & 17.3 & 1.80 & $* * *$ & $* * *$ \\
\hline FKM & $\mathrm{CO}$ & 11.0 & 1.85 & $* * *$ & $* * *$ \\
\hline NBR & $\mathrm{CO}$ & 18.9 & 1.06 & $* * *$ & $* * *$ \\
\hline SPA & $\mathrm{CO}$ & 8.18 & 1.90 & * & $* * *$ \\
\hline SPB & $\mathrm{CO}$ & 5.59 & 0.50 & $* * *$ & $* * *$ \\
\hline SSW & $\mathrm{CO}$ & 5.83 & 0.59 & $* *$ & $* * *$ \\
\hline Con & $\mathrm{CO}$ & 3.58 & 0.21 & n.a. & $* * *$ \\
\hline Con3 & $\mathrm{CO}$ & 6.20 & 0.74 & n.a. & $* * *$ \\
\hline UT2 & $\mathrm{H}_{2}$ & 79.3 & 6.03 & $* * *$ & $* * *$ \\
\hline UT4 & $\mathrm{H}_{2}$ & 194 & 18.3 & $* * *$ & $* * *$ \\
\hline Tex & $\mathrm{H}_{2}$ & 17.8 & 1.47 & $* * *$ & $* * *$ \\
\hline Tgr & $\mathrm{H}_{2}$ & 3.39 & 0.22 & $* * *$ & $* * *$ \\
\hline Tbl & $\mathrm{H}_{2}$ & 7.46 & 2.35 & * & $* *$ \\
\hline FKM & $\mathrm{H}_{2}$ & 4.84 & 2.17 & & * \\
\hline NBR & $\mathrm{H}_{2}$ & 4.83 & 1.45 & * & $* *$ \\
\hline SPA & $\mathrm{H}_{2}$ & 6.28 & 2.34 & & * \\
\hline SPB & $\mathrm{H}_{2}$ & 3.90 & 0.67 & $* *$ & $* * *$ \\
\hline SSW & $\mathrm{H}_{2}$ & 3.27 & 0.97 & & $* *$ \\
\hline Con & $\mathrm{H}_{2}$ & 1.53 & 0.13 & n.a. & $* * *$ \\
\hline Con3 & $\mathrm{H}_{2}$ & 4.80 & 0.67 & n.a. & $* * *$ \\
\hline
\end{tabular}

n.a.: not applicable 


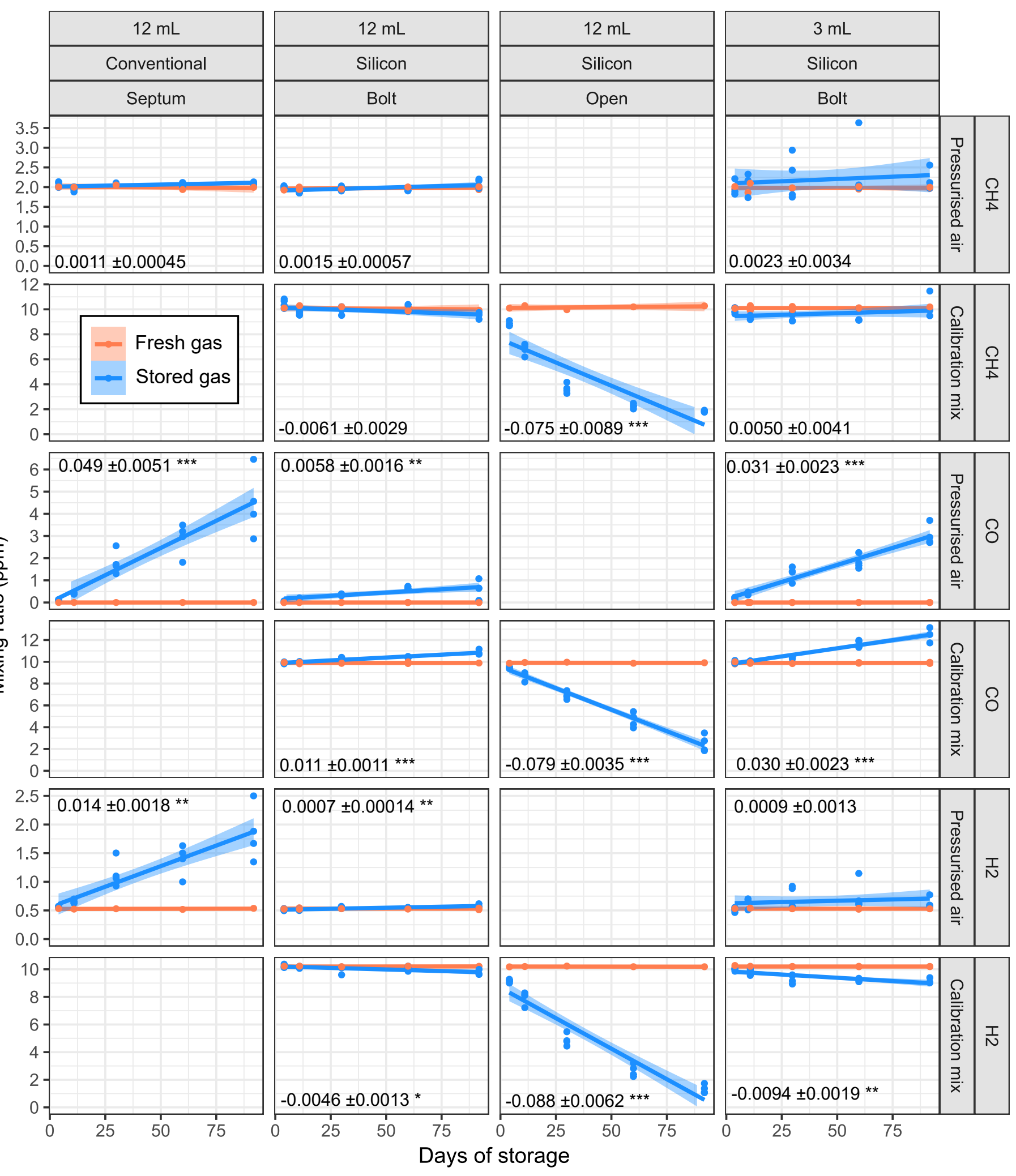

Fig. S1: Mixing ratios of stored gas samples and freshly prepared reference gas samples. The number listed is the slope \pm standard error of the stored gas mixing ratios calculated using linear regression with interactions; a significant difference to fresh gas is indicated with stars: ${ }^{* * *} p<0.001 ;{ }^{* *} p<0.01 ;{ }^{*} p<0.05$ 